\title{
Human Ranging of Aircraft: A Pilot Study
}

\author{
Kevin Rigby and Tanner Cheek \\ Embry-Riddle Aeronautical University
}

\begin{abstract}
The purpose of this research was to investigate the basic ability of humans to range in-flight aircraft. The question was posed during another research study by the author for the purpose of setting a quantitative baseline for automated sense-and-avoid distance. An experimental research design was used for the study. Aircraft position was based on reported Automated Dependent Surveillance Broadcast (ADS-B) data which is based on the Global Positioning System (GPS) fix of the aircraft. Humans in the pilot study ranged aircraft with a mean absolute error of 50.34\% at ranges between 650 and 9,738 meters.
\end{abstract}

\section{Introduction}

This pilot study examined the ability of human subjects to visually range in-flight aircraft. Unmanned systems are becoming more prevalent in our society, in the military, and in industry. Unmanned Aerial Systems (UAS) are commonplace in military operations. Limited commercial operations are allowed in the National Airspace System (NAS) on a case-by-case basis. One of the current Federal Aviation Administration (FAA) requirements placed on UAS operators in the NAS is sense-and-avoid capability (FAA, 2008). Humans and robots can sense using multiple complex systems. Previous work on human ranging of targets was well established as early as 1954, but was limited to human subjects ranging targets up to 400 yards (Gibson \& Bergman, 1954; Gibson, Bergman, \& Purdy, 1955; Purdy \& Gibson, 1955). Gibson and Bergman (1954) found that untrained subjects on a mowed grass field estimating ranges within 400 yards had an absolute error of between 7 and 20 percent.

A pilot flying a manned aircraft uses several methods to sense-and-avoid other aircraft. In reference to the sense-and-avoid principal, "A frequently asked question in human factors engineering is whether the role assigned to the human being is within his or her capabilities" (Liebowitz, 1988, p. 85). In both radar and non-radar environments visual scanning is the primary method used by pilots. Pilots must pick aircraft out of the visual field and determine whether an aircraft is a threat. Initial threat determination is based on whether or not the aircraft is on a collision course with the pilot's aircraft. Final threat determination is based on direction and velocity. Humans range objects using a combination of visual cues to include oculomotor cues (heuristic feelings in eye muscles, not possible beyond about 3 meters), pictorial cues (a pilot sees and identifies a Cessna 172 (C-172)), movement cues (a C-172 moves across a visual field at an estimable rate), and binocular disparity (the differences in scene between the left and right eye)(Goldstein, 1999). 


\section{Statement of the Problem}

UAS require sense and avoid capabilities for operation in the NAS. Below 10,000 feet in the NAS, aircraft are limited to 250 knots indicated airspeed (KIAS). At 250 KIAS, the closing rate is such that aircraft within 5 nautical miles (3.125 kilometers) of one another are considered a possible threat to each other. In terms of sense-and-avoid, if pilots can spot an aircraft in their visual field, they can then begin to determine threat by determining range.

Many manned aircraft carry transponders. However, many do not and are not required to do so by Federal Aviation Regulation (FAR). Systems like Terminal Collision Avoidance Systems (TCAS) and Automated Dependent Surveillance - Broadcast (ADSB) technologies are common in many new aircraft. However, they depend on other aircraft having transponders of one type or another. The threat of collision with a UAS is based on aircraft without transponders. These aircraft by FAR would be operating in Visual Meteorological Conditions (VMC). Therefore, human see-and-avoid is the primary current method of collision avoidance.

\section{Significance of the Study}

The study is significant in the fact that it establishes a quantitative baseline for human ability to range aircraft. This information is useful to both UAS and manned sense-and-avoid system developers.

\section{Review of Human Sensing}

The following review of human sensing gives an overview of human vision. It also establishes a rationale for the best case conditions for human visual sensing.

Humans use a variety of sensors to perceive the environment surrounding them and then recognize patterns that will produce a behavior. Behavior can be action or inaction. Human senses include visual (seeing), vestibular (inner ear), aural (hearing), taste, olfactory (smell), and tactile (touch). These senses are often combined into systems such as the somatosensory system, for example, which includes proprioception (the sense of position of the limbs) and kinesthesis (the sense of movement of the limbs) (Goldstein, 1999). The somatosensory system combines visual, vestibular, and kinesthetic sensors to achieve perception.

Humans use two senses for ranging of objects: aural and visual. For aural sensing, binaural cues (the differences between the left and right ear) result in interaural differences. Interaural time differences for example will give a cue of direction. Since pilots would be in an aircraft that interferes with these aural cues, aural ranging is not a variable in this study. This means that for detection and ranging of aircraft $100 \%$ of human sensing of in-flight aircraft will result from visual cues. 


\section{Visual Sensing}

Human visual sensing is based on the reception of visible light on the retina. Humans perceive visible light in the electromagnetic radiation spectrum in the range 380 to 760 nanometers in wavelength (DeHart, 1985). The retina is made up of an optical array of rod and cone shaped receptors. Photons excite the rods and cones and produce a stimulus. This stimulus is the result of light being transduced into electricity, a signal which is carried to the brain through the cerebral cortex. The pattern produced by the stimulus on the optical array results in a perceptual cue (Goldstein, 1999).

Rods and cones. The distribution of rods and cones in the eye is not even. The highest density of cones occurs near the center of the retina in an area about the size of this small letter "o" in a size 10 font (Goldstein, 1999). The fovea is the point of central focus of light through the lens of the eye. Outward from the fovea the distribution of rods and cones changes exponentially. Overall, the retina contains far more rods than cones, about 120 million rods and 6 million cones (Goldstein, 1999).

Rods are more sensitive to shorter wavelengths than cones. Cones receive peak light at a wavelength of 555 nanometers in the yellow-green spectrum. At about 510 nanometers rods begin to receive more light than cones and peak at around 490 nanometers in the blue-green spectrum. These differences in light cause differences in visual acuity as light changes. Visual acuity is highest in the cone rich fovea in bright light and shift to the rods as light diminishes until all luminosity is gone (DeHart, 1985). Based on this discussion, rods are more sensitive to light than cones due to the fact that they require less light. This means that movement of an object is more likely to be detected by the rods. This results in peripheral vision being more sensitive to movement. However, the cones are more sensitive to detail. Therefore, if fine movement is detected it must be targeted and directly viewed in the visual field. Direct viewing becomes more difficult as the light intensity drops.

Perceiving visual space. Humans perceive visual space using a combination of depth cues. "The cues approach to depth perception focuses on identifying information in the retinal image that is correlated with depth in the scene” (Goldstein, 1999,p. 215). There are two basic types of visual cues: oculomotor and visual. Oculomotor cues are cues which are kinesthetic. Visual cues are produced by the scene played out on the retina and are subdivided into monocular and binocular cues. Monocular cues include pictorial and movement-produced cues. Binocular cues are based in stereopsis (Goldstein, 1999; Blake \& Sekuler, 2006).

Oculomotor cues. Oculomotor cues are based on a human's ability to sense the position of our eyes and the tension in eye muscles. These cues are based on basic feelings in the eyes that occur from two sources, the eye muscles that move the eyes and from the movement of the lens of the eye. Convergence occurs when the eyes target something close to the face and the eyes cross, producing tension in the muscles of the 
eyes. This is a cue that the object is near. Accommodation occurs when the lens of the eye changes shape and bulges to focus on an object near the face (Goldstein, 1999; Blake \& Sekuler, 2006). Oculomotor cues are only reliable at a distance of about 1 to 3 meters and are not reliable cues in the detection of distant objects such as in-flight aircraft.

Pictorial cues. Pictorial cues are static depth cues that can be depicted in a painting by an artist or in a photograph (Goldstein, 1999; Gibb, Gray, \& Scharff, 2010). Making sense of pictorial cues is heuristic in nature, meaning that the observer must be able to identify objects in a scene and have some prior knowledge about those objects. Pictorial cues include: occlusion, atmospheric perspective, relative height, familiar size, linear perspective, texture gradient, and shadows. (Goldstein 1999; Gib et al., 2010).

Take for example, a flatland that leads to distant mountains. An occlusion would occur if one mountain partially hides another and an observer would know that the occluded mountain is farther away. If the sky were clear then an observer would be able to see more detail and the atmosphere would have an effect that would make the mountain seem to be nearer than if it were hazy (atmospheric perspective). If the mountain were near and the peak above the observer, then the object would appear higher in the visual scene and a sense of height would be gained (relative height). If a car were on the side of the mountain on a road, a sense of familiar size would be gained. If a straight road led to the mountain, and the lines of the road disappeared into the distance, then a sense of linear perspective would be gained. If a series of equally farmed fields were next to the road in the valley, and led up to the mountain, then a texture gradient would be evident, and the farther fields would appear smaller. If the sun were setting behind the mountains, then shadows would begin to fall in front of the mountain and provide more linear perspective. Using these pictorial cues an observer could make an estimation of range.

A pilot attempting to determine the range of an aircraft might use any of these visual cues to estimate range. The primary pictorial cue that may affect range estimation in aviation is atmospheric perspective, especially if there are no other visual cues in the sky. Haze in the atmosphere will reduce the visible detail of an aircraft. This might make the aircraft unrecognizable or seem slightly smaller. The pilot would merely know that an object is in the distant sky and range determination would be highly unreliable.

Movement-produced cues. An observer may move and the observed object may move. These movements produce two movement cues, motion parallax and deletion/accretion. Motion parallax is produced by the appearance of near or far objects appearing to move at relatively different rates across the visual field. Deletion/accretion occurs when two objects overlap and movement covers (deletion) or uncovers (accretion) the object which is more distant. Deletion/accretion is related to motion parallax in that the overlapping surfaces appear to move relative to one another. Deletion/accretion is related to the pictorial cue of occlusion (Gibb et al, 2010). An object that moves faster in 
the visual field will appear nearer than an object that moves slower. (Goldstein, 1999; Gibb et al., 2010).

Take for example a driver speeding down a road in an open field who enters a segment of road lined with evenly spaced trees. The trees in the distance will appear to move more slowly than the trees that are near due to motion parallax. Accretion is also occurring as distant trees are uncovered. Depending on where the driver looks, his/her sense of speed will change. If the driver enters another segment of road where the trees are at twice the distance from the road, then a variable in the motion cue has changed and the driver may experience a difference in perceived distance. Other variables that would affect the perceived cue might be the type, size, and spacing of the trees. Atmospheric perspective will also affect motion parallax by reducing the detail of the trees making them appear smaller and spaced further apart.

Motion processing. The object moving in the visual field will cause a local shift of an image on the retina. An observer moving the eyes or the body will cause an entire shift of the visual image on the retina. "Expansion, contraction, and rotation of the entire visual field are all components of optical flow information” (Gibb et al., 2010, p. 45). Optical flow is another term for motion parallax (Davis, Johnson, Stepanek, \& Fogarty, 2008).

Binocular disparity. Stereoscopic vision is based in binocular disparity. "Stereoscopic vision involves combining the images from the two eyes in order to judge the depth of objects in one's environment" (Gibb et al., 2010). Binocular disparity is based on the differences between the scenes presented to the optical matrix of the retina. Retinal disparity is the difference between the location of an object on a given plane in the two separate scenes, or images (Blake \& Sekuler, 2006). The appearance of the model aircraft in Figure 1 is an example of disparity between a left and right camera image at a range of approximately 1 meter and a baseline of approximately 0.1 meter.

In essence, each eye gives a different viewpoint of a viewed object (Goldstein, 1999). Simply closing one's eyes alternately, while focusing on an object, will create the effect. The magnitude of disparity is a function of how far away the object is and how far apart the eyes are located. Binocular disparity $(\delta)$ is related to depth $(\Delta \mathrm{D})$, interocular separation (I), and distance (D) as seen in Equation 1 (Gib et al.):

$$
\delta \approx \mathrm{I} \Delta \mathrm{D} / \mathrm{D}^{2}
$$

Binocular disparity will change with the square of the distance and become very small as distance increases. In humans, interocular separation can be assumed at approximately 65 millimeters and will not vary more than a few millimeters in a normal adult (Hibbard, 2008). 
Rearranging Equation 1 results in Equation 2:

$$
\Delta \mathrm{D}=\delta \mathrm{D}^{2} / \mathrm{I}
$$

Figure 2 is an example of how distance affects the relative disparity of an object when viewed from 1 and 5 meters on a 0.065 meter baseline from an Olympus FE-230 point and shoot CCD camera.

Environmental variables affecting pilots. Atmospheric perspective and low luminosity have already been discussed as environmental variables that can affect the variability of depth perception in humans. Environmental variables that specifically affect pilots include vibration, hypoxia, visual acuity, and contaminated windscreens.

Vibration directly affects the lens of the eye. A large range of vibrations are transmissible to the pilot in an aircraft (Dehart \& Davis, 2002). "Difficulties in reading instruments and performing visual searches occur when vibrations introduce relative movement of the eye with respect to the observed object or target" (DeHart \& Davis, 2002, p. 165).

Hypoxic (altitude) hypoxia occurs in pilots as altitude is increased. As altitude increases, the density of the air humans breathe decreases. Therefore, the amount of oxygen per breath decreases. This reduction of oxygen results in lower blood oxygenation, and has adverse effects in humans. The symptoms of hypoxic hypoxia become evident after about 5,000 feet above mean sea level (MSL) (Reinhart, 2008). "Vision is the first of the special senses to be altered by a lack of oxygen, as evidenced by diminished night vision” (DeHart \& Davis, 2002, p. 368).

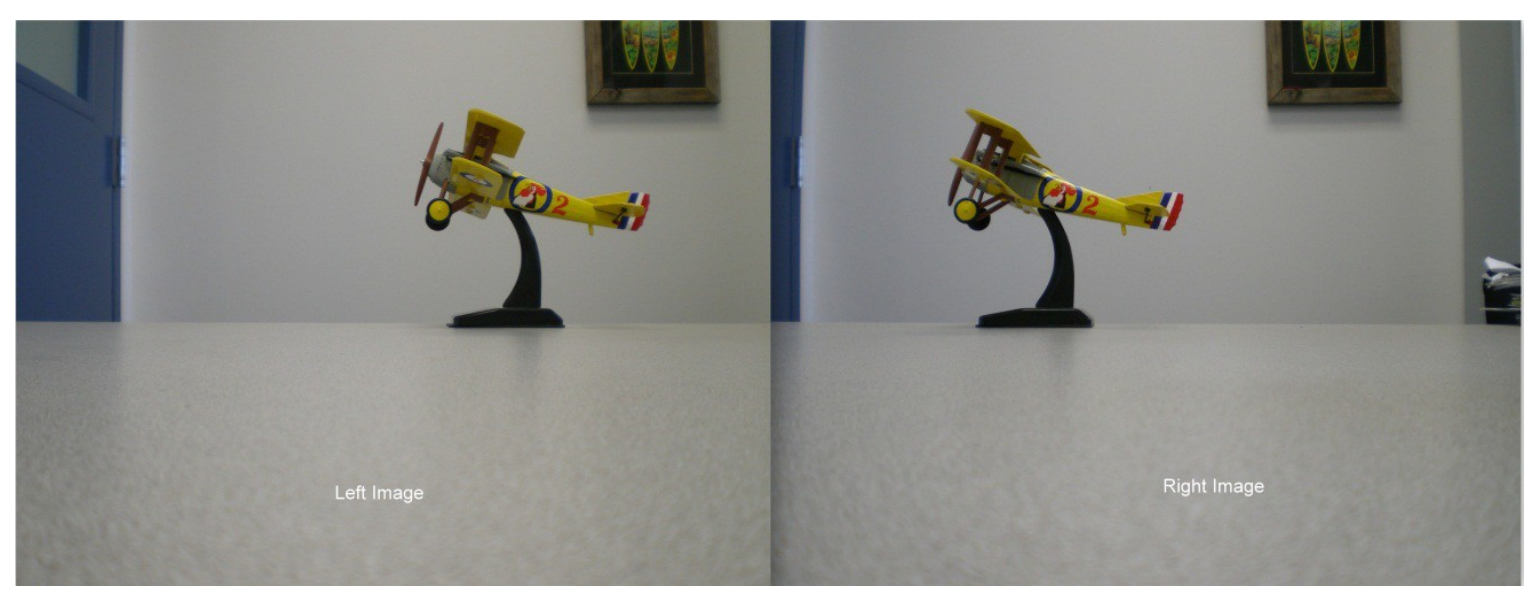

Figure 1. Narrow base-line disparity. 


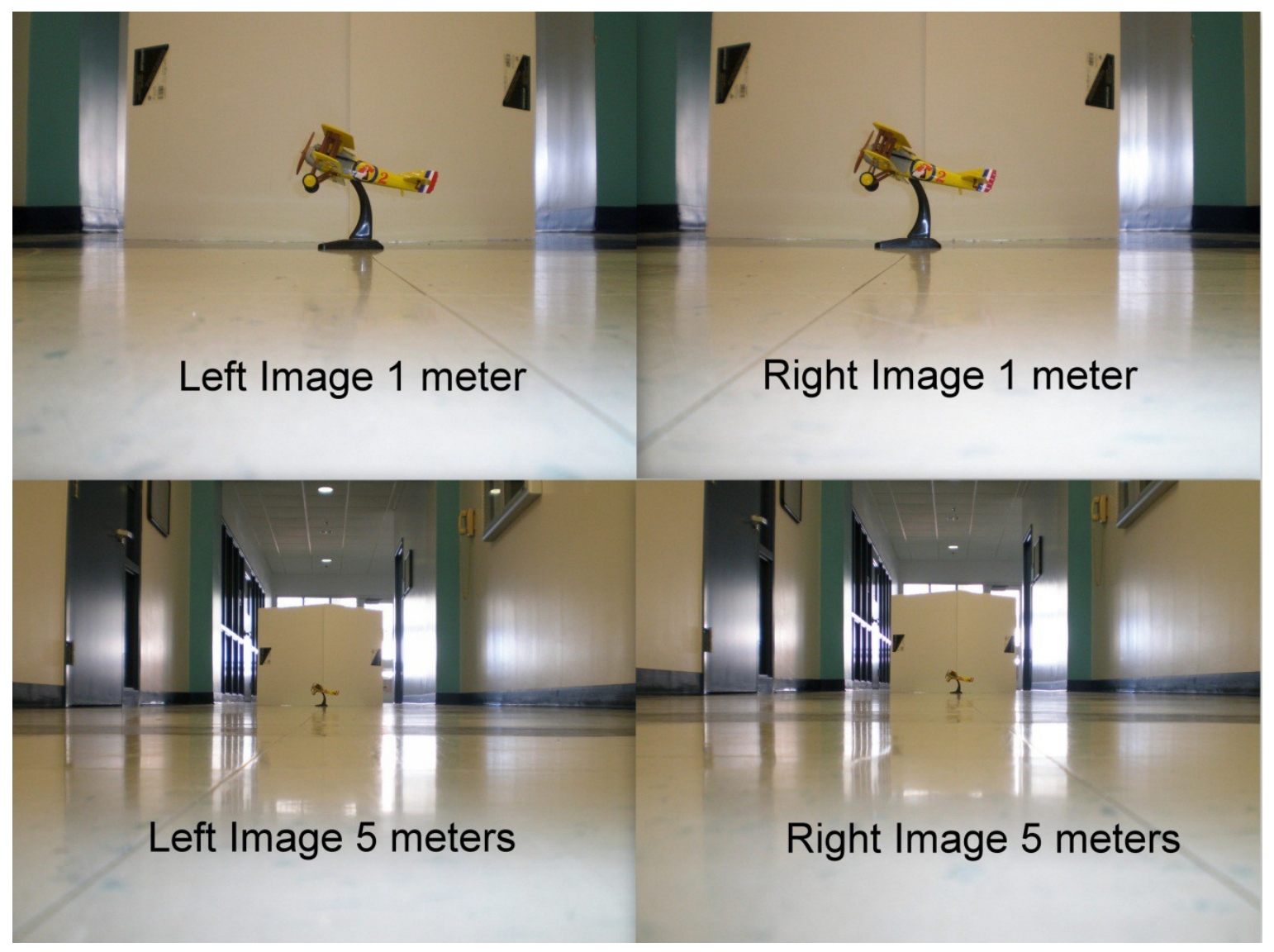

Figure 2. Relative disparity at 1 and 5 meters using a human equivalent baseline of 0.065 meter.

As distance increases to very large ranges such as between two aircraft, small changes in disparity serve as a poor cue for depth perception and it is assumed that pilots will rely primarily on monocular cues (Gibb et al., 2010). According to Goldstein (1999), binocular disparity cues become unreliable at about 30 meters.

In private pilots, visual acuity would serve as a variable in visual sensing. Private pilots are required by the FAA to hold a $3^{\text {rd }}$ Class medical certificate. Vision requirement to obtain the $3^{\text {rd }}$ Class medical certificate is $20 / 40$ or better visual acuity in each eye with or without correction (FAA, 2010). Visual acuity is how sharp or crisp an object will appear to be at a given distance. Normal visual acuity is 20/20 and is tested for example by a subject being able to read a given line of letters on a chart at 6 meters distance (DeHart, 1985). Anything greater than 20/20, 20/40 for example, means that the subject will not have the same clarity of a visual image as a person with 20/20 visual acuity. This difference in visual acuity will affect the variability of depth perception in the same way as atmospheric perspective by reducing detail and reliability of pictorial cues. A film of 
dirt on a windscreen might have a similar effect.

\section{Summary}

Humans visually sense based on the reception of visible light. Visual space is perceived based on several depth cues to include both monocular and binocular cues. At ranges beyond about 30 meters binocular cues become unreliable and monocular cues are primary for depth perception. Visual sensing reliability diminishes as environmental factors involved in aviation are considered. For the purpose of this research, the best case scenario for perceiving visual space would be a person standing on the ground at sea level near noon on a clear day. The worst case scenario would be a private pilot with 20/40 visual acuity in an aircraft with high vibration and a dirty windscreen flying at sunrise or sunset above 5,000 feet MSL. The best case scenario will be considered for this research as it would be the minimum error encountered. The only variable not accounted for in the pilot study on human ranging was that of acuity.

\section{Method}

The following will present the research design, procedures, and data collection techniques that were used in this study. The technologies used in this study will also be discussed.

\section{Research Design}

The research design used in this study was a quantitative experimental method. The samples were completely self-selected in the sense that the experimenter had no control over the subjects involved in the human ranging pilot study or the aircraft involved in the stereo ranging study.

\section{Samples}

Human subjects were taken from the random population of students who walked by the experimental area. Aircraft samples were taken from in-flight aircraft within visible range and field of view of the subject.

\section{Variables}

The independent variable in both the human range pilot study and the stereo range study that required precision was that of Global Positioning System (GPS) determined position of the aircraft. The method of record for the study was that of ADS-B reported position. ERAU fleet Cessna 172 aircraft are equipped with identical Garmin G1000 integrated ADS-B glass cockpits.

There were two independent variables in the human ranging pilot study. The first 
independent variable was the GPS determined position of the aircraft. The second independent variable was the human subject estimated range of the aircraft.

Independent variable 1. GPS determined aircraft position was the first independent variable. In this study, GPS position was determined using ADS-B reported data. The ADS-B data was taken from a program developed by the ERAU NEAR Lab that continuously logs ADS-B data. Units of measure were recorded in kilometers and converted to meters for final reporting and analysis.

Independent variable 2. Human subject estimated range was determined by the subject. Each subject was asked to pick an aircraft visible to them and within their field of view and state the range of the aircraft using the unit of measure that they felt most comfortable using. All units of measure were converted to meters for reporting and analysis.

\section{Procedure}

The following procedure outlines the experimental setup of the human range pilot study. The experiment took place at ERAU Daytona Beach under VMC with a METAR reported visibility of greater than 6 nautical miles. Only ADS-B reporting aircraft were included in the study. To ensure consistency, only ERAU ADS-B equipped Cessna 172 fleet aircraft were included in the data.

Location setup. A table was set up in an area with high student traffic. The area had a 360 degree lateral view of the sky and between 3 and 30 degrees vertical view in reference to the horizon.

Data collection. Two sources of data were recorded in this study, ADS-B data and subject reported data. The ADS-B data was recorded on an ERAU server. The data was provided to the researcher in a comma separated variable (.CSV) format. The subject reported data was hand recorded.

Subject reported data. Subjects verbally announced the estimated range of an aircraft. The subject estimated range was then recorded along with time, researcher estimated range, researcher estimated altitude, and magnetic bearing to the aircraft. Magnetic bearing was determined using a lensatic compass.

ADS-B data. The ERAU ADS-B database was queried for ADS-B data for the time duration of the experiment. The researcher filtered the data to a radius of 15 kilometers. The researcher was then able to identify the aircraft in the database data-set based on time, bearing, and altitude when compared to the recorded subject data. No other aircraft were in the vicinity of the viewing field when a subject observed the aircraft. 


\section{Results}

A total of 31 subjects participated in the human ranging pilot study. Seven of the aircraft targets did not have ADS-B onboard, so seven of the data points were not usable. Therefore, a total of 24 data points were analyzed. There were two extreme outliers that were outside of two standard deviations. The outliers were removed from the data leaving 22 total data points that are included in the analysis of the ranging study. The aircraft ADS-B positions ranged from 650.6 to 9,738.3 meters. The 22 subjects' estimations ranged varied from 24 to 11,265 meters. The absolute percent error was calculated for each pairing between the ADS-B position and the estimated range. The mean absolute percent error was 50.34\%. Appendix A presents the post-processed human ranging pilot study data.

Table 1

Human Ranging Pilot Study Descriptive Data

\begin{tabular}{lll}
\hline Data Type & ADS-B Position & Subject Estimated Range \\
\hline$n n$ & 22 & 22 \\
Mean & $3406.3 \mathrm{~m}$ & $1691.7 \mathrm{~m}$ \\
Standard Deviation & $2780.6 \mathrm{~m}$ & $2504.5 \mathrm{~m}$ \\
High & $9738.3 \mathrm{~m}$ & $11265.0 \mathrm{~m}$ \\
Low & $\underline{650.6 \mathrm{~m}}$ & $24.0 \mathrm{~m}$ \\
\hline
\end{tabular}

\section{Discussion}

The study was completed as a pilot study. The limitations and recommendations identified in this discussion should be considered for future studies.

\section{Limitations of the Study}

Limitations of the study include the following:

No demographic data on the subjects was recorded.

1. No information on aviation experience was recorded.

2. No information on visual acuity was recorded.

3. Only one type of aircraft was used in the study.

4. The study was performed on a single day with no variation in meteorological conditions.

5. No localization information was asked of the subjects.

No identifying data was collected due to the fact that this study was time sensitive as an addition to another primary study in stereo ranging of aircraft using wide-baseline stereopsis. The question was posed during the stereopsis research as to how well humans can range in-flight aircraft, and a review of the literature resulted in no specific works on 
the topic. Internal review board (IRB) approval would have been required for collecting identifying data of the human subjects, and the time frame to do so did not fit that of the stereopsis research.

\section{Recommendations for Future Research}

Recommendations for this study include the use of aircraft capable of logging GPS position and a full scale expansion of the ranging pilot study.

1. Replicate the study using Cessna 172 (C-172) aircraft equipped with the Garmin FDM capable of logging the GPS position of the aircraft at $10 \mathrm{hz}$.

2. Replicate the study under varying meteorological conditions.

3. Perform a scale version of the human ranging study to include localization.

4. Collect data on pilots versus non-pilots experience level.

5. Collect demographic data on each subject.

6. Test visual acuity of each subject.

Replicating the study with a Garmin FDM would allow the researcher to gain a more precise position of the aircraft at the time of observation. It also allows the researcher to gather localization data about the aircraft. Localization data includes altitude, rate of climb, and heading in addition to the latitude and longitude of the aircraft. These are variables that are used to determine threat in the pilot see-and-avoid process.

Variations in meteorological conditions would be a positive addition to the study. Visibility would be the primary factor due to the effect it may have on aircraft detail (atmospheric perspective) presented to the naked eye.

\section{Conclusion}

The question of how well humans range in-flight aircraft was very broad in nature. The main purpose of this study was to determine an initial baseline for human ranging of in-flight aircraft for the stereopsis research being performed by the primary researcher in sense-and-avoid for UAS. This paper provided a discussion of the method, results, limitations, and recommendations of the study. The results provide data that suggests that humans have a very large error in estimating the range of in-flight aircraft. Further research is needed in a full scale study to determine the actual error. 


\section{References}

Blake, R., \& Sekuler, R. (2006). Perception (5 ${ }^{\text {th }}$ ed.). New York, NY: McGraw-Hill.

Davis, J.R., Johnson, J., Stepanek, J., \& Fogarty, J.A. (Eds.). (2008). Fundamentals of Aerospace Medicine ( $4^{\text {th }}$ ed.). Philadelphia, PA: Lippincott Williams \& Wilkins.

Dehart, R.L. (Ed.). (1985). Fundamentals of Aerospace Medicine. Philadelphia, PA:

Lea \& Febiger.

Dehart, R.L., \& Davis, J.R. (Eds.). (2002). Fundamentals of Aerospace Medicine. (3 ${ }^{\text {rd }}$ ed.). Philadelphia, PA: Lippincott Williams \& Wilkins.

Federal Aviation Administration. (2008). Interim Operational Approval Guidance 08-01. Washington, DC: Author.

Federal Aviation Administration. (2010). Federal Aviation Regulations (Part 67). Washington, DC: Author,

Gibb, R., Gray, R., \& Scharff, L. (2010). Aviation Visual Perception. Burlington, VT: Ashgate Publishing Company.

Gibson, E.J., \& Bergman, R. (1954). The effect of training on absolute estimation of distance over the ground. Journal of Experimental Psychology, 48(6), 473-482.

Gibson, E.J., Bergman, R., \& Purdy, J. (1955). The effect of prior training with scale of distance on absolute and relative judgments of distance over ground. Journal of Experimental Psychology, 50(2), 97-105.

Goldstein, E.B., (1999). Sensation and perception (5 ${ }^{\text {th }}$ ed.). Pacific Grove, CA: Brooks/Cole Publishing Company.

Hibbard, P.B. (2008). Binocular energy responses to natural images. Vision Research, 48(1), 1427-1439.

Liebowitz, H. W. (1988). The human senses in flight. In E. L. Wiener \& D.C. Naagel (Eds.), Human factors in aviation (pp. 83-110). New York, NY: Academic Press.

Purdy, J. \& Gibson, E.J. (1955). Distance judgment by the method of fractionation. Journal of Experimental Psychology, 50(2), 374-380.

Reinhardt, R.O., (2008). Basic Flight Physiology. New York, NY: McGrawHill. 


\section{Appendix A}

\section{Human Ranging Pilot Study Data}

\begin{tabular}{|c|c|c|c|c|c|c|c|c|c|}
\hline Set & $\begin{array}{c}\text { Target } \\
\text { Address }\end{array}$ & $\begin{array}{l}\text { Meters } \\
\text { ADS-B }\end{array}$ & $\begin{array}{l}\text { Bearing } \\
\text { ADS-B }\end{array}$ & Time ADS-B & EST Time & $\begin{array}{l}\text { Est. } \\
\text { Bearing }\end{array}$ & $\begin{array}{l}\text { Est. } \\
\text { Meters }\end{array}$ & \%Error & $\begin{array}{l}\text { \% Error } \\
\text { Absolute }\end{array}$ \\
\hline 1 & 10845621 & 650.57 & 25.65 & 18.8598329 & 18.879 & 30 & 400 & -38.515 & 38.515 \\
\hline 2 & 10779949 & 865.30 & 54.27 & 19.45060981 & 19.46 & 40 & 610 & -29.504 & 29.504 \\
\hline 3 & 10832657 & 925.62 & 163.63 & 19.03388889 & 19.036 & 150 & 244 & -73.639 & 73.639 \\
\hline 4 & 11098297 & 938.23 & 156.00 & 19.10411024 & 19.108 & 140 & 1931 & 105.814 & 105.814 \\
\hline 5 & 11112212 & 1056.01 & 68.97 & 18.91838759 & 18.922 & 60 & 300 & -71.591 & 71.591 \\
\hline 6 & 10854781 & 1154.66 & 135.17 & 19.5996658 & 19.604 & 160 & 644 & -44.226 & 44.226 \\
\hline 7 & 10886065 & 1467.70 & 108.99 & 18.35688802 & 18.367 & 100 & 402 & -72.610 & 72.610 \\
\hline 8 & 10888918 & 1758.29 & 100.02 & 18.58272135 & 18.588 & 130 & 100 & -94.313 & 94.313 \\
\hline 9 & 11109359 & 1784.23 & 99.32 & 19.34210938 & 19.347 & 100 & 2414 & 35.297 & 35.297 \\
\hline 10 & 11098297 & 2036.71 & 175.70 & 19.27333333 & 19.246 & 160 & 1207 & -40.738 & 40.738 \\
\hline 11 & 11098297 & 2077.47 & 103.63 & 19.1146658 & 19.119 & 110 & 4023 & 93.649 & 93.649 \\
\hline 12 & 11098297 & 2127.90 & 101.72 & 19.32866536 & 19.3 & 140 & 600 & -71.803 & 71.803 \\
\hline 13 & 10761279 & 2441.08 & 110.13 & 18.4573329 & 18.479 & 123 & 1979 & -18.929 & 18.929 \\
\hline 14 & 10761279 & 3238.57 & 153.92 & 18.3818316 & 18.4 & 160 & 4023 & 24.221 & 24.221 \\
\hline 15 & 10834559 & 3414.68 & 152.05 & 18.98294271 & 19 & 140 & 549 & -83.922 & 83.922 \\
\hline 16 & 10863941 & 4885.51 & 10.27 & 19.29405382 & 19.297 & 10 & 4023 & -17.654 & 17.654 \\
\hline 17 & 10832657 & 5378.40 & 97.92 & 19.07116536 & 19.092 & 70 & 305 & -94.329 & 94.329 \\
\hline 18 & 10852879 & 6331.40 & 102.84 & 18.68255425 & 18.683 & 120 & 1200 & -81.047 & 81.047 \\
\hline 19 & 11109359 & 6521.37 & 149.54 & 19.3971658 & 19.404 & 140 & 61 & -99.065 & 99.065 \\
\hline 20 & 10832657 & 7039.47 & 103.01 & 19.08399957 & 19.097 & 100 & 914 & -87.016 & 87.016 \\
\hline 21 & 10501486 & 9107.41 & 246.76 & 19.42861111 & 19.433 & 210 & 11265 & 23.690 & 23.690 \\
\hline 22 & 11404308 & 9738.26 & 170.65 & 19.01822483 & 19.008 & 170 & 24 & -99.754 & 99.754 \\
\hline
\end{tabular}

